Original Research Paper

\title{
Safety Assessment of Indigenous Probiotic Strain Lactobacillus plantarum Dad-13 Isolated from Dadih Using Sprague Dawley Rats as a Model
}

\author{
1,5Endang S Rahayu, ${ }^{2}$ Ilzamha H Rusdan, ${ }^{3}$ Armita Athennia, ${ }^{1}$ Rafli Z Kamil, \\ ${ }^{1}$ Putrika C Pramesi, ${ }^{1}$ Yustinus Marsono, ${ }^{1}$ Tyas Utami and ${ }^{4}$ Jaka Widada \\ ${ }^{1}$ Department of Food and Agricultural Product Technology, \\ Faculty of Agricultural Technology, Universitas Gadjah Mada, Yogyakarta, Indonesia \\ ${ }^{2}$ Institut Agama Islam Negeri Surakarta, Indonesia \\ ${ }^{3}$ Department of Nutrition, Universitas Muhammad Husni Thamrin, Jakarta, Indonesia \\ ${ }^{4}$ Department of Microbiology, Faculty of Agricultural, Universitas Gadjah Mada, Yogyakarta, Indonesia \\ ${ }^{5}$ Center for Food and Nutrition Studies, Universitas Gadjah Mada, Yogyakarta, Indonesia
}

\author{
Article history \\ Received: 19-08-2019 \\ Revised: 19-10-2019 \\ Accepted: 11-11-2019 \\ Corresponding Author: \\ Endang S. Rahayu \\ Department of Food and \\ Agricultural Product \\ Technology, Faculty of \\ Agricultural Technology, \\ Universitas Gadjah Mada, \\ Yogyakarta, Indonesia \\ Email: endangsrahayu@ugm.ac.id
}

\begin{abstract}
Lactobacillus plantarum Dad-13 is a probiotic candidate from dadih, a traditional fermented buffalo milk from West Sumatera, Indonesia. This study aimed to evaluate safety aspects of L. plantarum Dad-13 in high dose consumption on Sprague Dawley rats. Two treatment groups were fed with $10^{11} \mathrm{CFU} / \mathrm{mL} /$ day of $L$. plantarum Dad-13 for 14 and 28 days respectively. Two placebo groups were fed with $1 \mathrm{~mL}$ skimmed milk per day for 14 and 28 days and a fifth, untreated group used as control. Feed intake and body weight were monitored, while blood samples and mesenteric lymph node (MPN) organs were dissected. Organ weight, leukocyte profiles, glutamic-oxaloacetic transaminase (GOT) activity, plasma malondialdehyde (MDA) concentration and intestinal morphology were measured. Microbial analyses were conducted on fecal matter, digesta, blood and organs. Results showed that consumption did not negatively affect general health, organ weight, leukocyte profiles, GOT activity, MDA concentration and intestinal morphology. Numbers of $L$. plantarum in treated rats' feces were significantly increased, indicating its survival in gastrointestinal tracts. Bacteria in the blood and organs of both groups were identified using rep-PCR with BOX A1R primer, which revealed that it was not identical to L. plantarum Dad-13. Thus, L. plantarum Dad-13 did not translocate in the organs and blood of rats. Therefore, $L$. plantarum Dad-13 is suggested as likely to be safe for human consumption.
\end{abstract}

Keywords: Safety Assessment, Probiotic, Bacterial Translocation

\section{Introduction}

Probiotics are live organisms which, when consumed in sufficient amounts, confer beneficial effects on the host (FAO/WHO, 2002). Probiotic organisms commonly belong to the genera of Lactobacillus and Bifidobacterium. Many probiotics have their origins in dairy products and fermented foods. For many years they have been consumed as components of these foods without any apparent negative effects on the health of the consumer. In the production of traditional foods, lactic acid bacteria have been accepted as safe in the absence of scientific criteria, because they are micro-flora which is normally found in the intestinal tract and have a long history of use devoid of adverse effects (Donohue, 2006).

In many traditional Indonesian fermented foods, lactic acid bacteria are recognized components, most of which occur as spontaneous fermentations. Lactobacillus plantarum is one of the most common types of lactic acid bacteria found in traditional Indonesian fermented food (Rahayu, 2003). It is an indigenous lactic acid bacterium isolated from dadih, a traditional fermented buffalo milk from West Sumatera, Indonesia. This bacteria type has met the basic requirements for a 
probiotic and has some functional properties. Consumption of fermented milk containing L. plantarum Dad-13 by thirty healthy Indonesian volunteers resulted in a significant increase in the numbers of L. plantarum in the fecal matter of all volunteers and a decrease in the amounts of Enterobacteriaceae, E. coli and coliform non $E$. coli in the fecal matter of more than $50 \%$ of the volunteers (Rahayu et al., 2016). This indigenous probiotic can also be used as a starter culture for milk fermentation (Wardani et al., 2017).

Although L. plantarum Dad-13 was isolated from traditional fermented buffalo milk that has been consumed for many years without adverse effects, the safety aspect of long-term consumption of this bacteria type had not yet been studied. The safety of new probiotic strains needs to be studied since probiotic bacteria are strain-specific (Salminen et al., 1998). A study evaluated the safety aspects of probiotic strains using animal models in which a safety assessment for Lactobacillus salivarius CECT5713, Lactobacillus rhamnosus HN001 and Lactobacillus acidophilus HN017 were carried out by orally introducing these bacteria in doses of $10^{10} \mathrm{CFU} / \mathrm{rat} /$ day for 28 days (Shu et al., 2000a). Safety evaluation was carried out by monitoring the viability of the rats, body weight changes, feed intake, biochemical analyses of blood and microbial analyses of organ samples of the rats. The results showed that there were no significant effects due to the consumption of those bacteria on the treated rats and thus probiotics were judged safe to be consumed. Other safety parameters for probiotic bacteria are the ability of the bacteria to translocate to blood and organs, to produce metabolites that affect gastro-intestinal tract and having a transfer gene that is resistant to antibiotics. Disease occurrence such as bacteremia and fungemia caused by Lactobacilli can be considered as one parameter to assess the safety aspects of probiotic (Snydman, 2008). In year 1989 to 2004, 86 cases of Lactobacillus bacteremia were identified, but only one case of L. plantarum was found in the blood sample of a patient (Salminen et al., 2006). Previous studies found that bacteremia and fungemia occurrence related to the use of probiotic is rare (Snydman, 2008).

Probiotic effects are strain-specific. Thus, so safety assessments need to be carried out on a strain by strain basis. The aim of this study, therefore, was to evaluate the safety of L. plantarum Dad-13, using Sprague Dawley rats as a model by orally introducing high doses (10 $\left.{ }^{11} \mathrm{CFU}\right)$ of L. plantarum Dad-13. Some parameters were analyzed including feed intake, body weight change, organ weight, total and profile leukocyte, Malondialdehyde (MDA) levels, Glutamic-Oxaloacetic Transaminase (GOT) enzyme activities and gut morphology. The possibility of translocation of $L$. plantarum Dad-13 to the blood and organs of the rats was also investigated.

\section{Materials and Methods}

\section{Bacterial Strain and Cell Production}

Lactobacillus plantarum Dad-13 was obtained from the Food and Nutrition Culture Collection (FNCC) Center for Food and Nutrition Studies, Universitas Gadjah Mada, Yogyakarta, Indonesia. Biomass production was carried out by inoculation of $10 \mathrm{~mL} 24 \mathrm{~h} \mathrm{~L}$. plantarum Dad-13 into $1 \mathrm{~L}$ of Peptone Glucose Yeast extract broth and incubated at $37^{\circ} \mathrm{C}$ for $24 \mathrm{~h}$. The culture was then cold centrifuged for 20 minutes at $3000 \mathrm{rpm}$ and the pellet was re-suspended in $10 \mathrm{~mL}$ of $10 \%$ skimmed-milk solution. The biomass was enumerated and stored at $-20^{\circ} \mathrm{C}$.

\section{Experimental Design}

Within this research, rats were used as research subjects with adherence to the Declaration of Helsinki guidelines. The research had been approved by the Medical and Health Research Ethics Committee (MHREC) according to a letter bearing the reference number KE/FK/193/EC and dated 18 March 2014.

Twenty male Sprague Dawley rats aged between 6 and 8 weeks were fed with AIN-93M diet and water ad libitum for a week as an adaptation phase (Reeves et al., 2018). Each rat was housed into one cage, separate from each other. The rats were then divided into five groups of four. The first and second groups were treated with $1 \mathrm{~mL}$ of $10 \%$ skimmed-milk containing $10^{11} \mathrm{CFU}$ L. plantarum Dad-13 each day for 14 and 28 days respectively and defined as the treatment groups. The intervention was done by force feeding. The third and fourth groups were fed with $1 \mathrm{~mL}$ of $10 \%$ skimmed-milk for 14 and 28 days respectively, named as placebo groups. The fifth group, called the control group, did not receive any treatment. Feed intake and body weight of the rats were recorded every day. After 14 and 28 day of treatment for the four test groups and after adaptation for the control group, all rats were euthanized with chloroform. Subsequently, blood samples and mesenteric lymph node (MPN) organs i.e. kidneys, livers and spleens were dissected and weighed aseptically.

\section{Biochemical, Hematological and Gut Histological Analyses}

Leukocyte profiles were analyzed using a hematology counter with fresh blood. Glutamic-Oxaloacetic Transaminase (GOT) enzyme activity in plasma samples was measured by DiasSys kit and absorbance was measured using a spectrophotometer at $365 \mathrm{~nm}$. Malondialdehyde levels were measured by thiobarbituric assay procedure (Albro et al., 1986). Ileum, caecum and colon morphologies were analyzed qualitatively using a microscope and quantitatively by 
measuring epithelial height, villous height and mucosa thickness. The tissue samples were stained using hematoxylin eosin (Shu et al., 2000a).

\section{Microbial Analyses of Fecal Matter, Digesta, Blood and Organs in Rats}

Fecal matter and digesta samples ( 1 g) were homogenized and diluted by serial dilution with a saline phosphate buffer $(9 \mathrm{~mL}) .100 \mu \mathrm{L}$ of each sample was inoculated in MRS and L. plantarum Selective Medium (LPSM) by spread plate method and incubated at $37^{\circ} \mathrm{C}$ for $48 \mathrm{~h}$. Colonies were enumerated, using a Quebec colony Counter, as lactic acid bacteria and L. plantarum respectively (Bujalance et al., 2006).

To evaluate possible bacterial translocation to the blood and organs of the rats, a $0.1 \mathrm{~mL}$ sample from the liver, kidneys, spleen and blood of each rat was introduced into test tubes containing MRS broth and then incubated at $37^{\circ} \mathrm{C}$ for $24 \mathrm{~h}$ for enrichment. Each sample was then inoculated into MRS and LPSM agar using streak plate method until a single colony was obtained. Colonies appearing in MRS and LPSM agar were suspected to be lactic acid bacteria and L. plantarum respectively and were tested for cell morphology and Gram stain ((Shu et al., 2000b) with modification).

\section{Identification of Isolated Bacteria using $16 \mathrm{~S}$ rRNA Gene Sequencing}

Each single colony from the blood and organs of rats that had morphology characteristics similar to $L$. plantarum in LPSM was grown in MRS broth. The next step was isolation of the DNA genome and purification of the DNA of the bacteria suspected to be L. plantarum, followed by amplification by rep-PCR primer BOXA1R (5'CTACGGCAAGGCAAGGCGACGCTGACGCTGA CG-3') and later followed by electrophoresis of the DNA fragments. DNA from pure L. plantarum Dad-13 was used as a reference and was compared to the isolates obtained from the blood and organs of treated and placebo rats to obtain fingerprints that would be used to develop dendogram similarities.

Total DNA genomes were isolated and purified ((Ausubel et al., 2002) with modification). The products coming from the PCR reaction were visualized with ethidium bromide staining on the agarose gel, $2 \%$ soaked with $100 \mathrm{~mL} 0.5 \mathrm{x}$ TBE at 100 volts for $30 \mathrm{~min}$. The visible rep-PCR pattern was then compared to an L. plantarum Dad-13 pattern. The next step was sequencing 16s rRNA of L plantarum Dad-13 and the suspected L. plantarum from the blood and organs of the rats, followed by analyzing the nitrogen base sequence and comparing it with the DNA nitrogen base sequence from gene bank.

\section{Result and Discussion}

\section{General Health Indicator and Biochemical and Hematological Parameters}

Oral administration by force feeding of high doses of L. plantarum Dad-13 did not make any significant differences to feed intake or body weight of either placebo or treatment groups. After 14 and 28 days the average body weight of all groups increased by about 20 $\mathrm{g}$ and $50 \mathrm{~g}$ respectively.

Biochemical and hematological analyses showed that there were no significant differences in leukocyte profiles and leukocyte totals between control and treatment groups $(\mathrm{p}>0.05)$. Hematology parameters, such as white blood cell measurement, are effective indicators in the detection of bacterial infection. Treatment with high doses of L. plantarum Dad-13 did not increase leukocyte profiles (Table 1) and leukocyte totals of the rats (Fig. 1). Significant increases in white blood cell levels could indicate an immune reaction to pathogens. Monocytes play a critical role in the prevention of intestinal pathogens by releasing macrophage, while neutrophil is responsible for phagocytosing microbes and producing antimicrobial factors (Kinnebrew and Pamer, 2012).

Oral treatment of high doses of L. plantarum Dad13 had no effect on biochemical parameters, such as malondialdehyde concentration and GOT enzyme activities. As shown in Fig. 2, there were no significant differences among any of the groups ((p>0.05). Consumption of $10^{10} \mathrm{CFU} /$ day/rat of $L$. salivarius CECT5713 showed GOT enzyme activities of $184.3 \mathrm{IU} / \mathrm{L}$ and $199.8 \mathrm{IU} / \mathrm{L}$ for control and treatment groups, respectively (Lara-Villoslada et al., 2007). GOT enzyme is a physiological change marker. GOT enzymes exist not only in the liver but also in other organ tissues. Excessive activity of this enzyme could be an indicator of organ damage, while malondialdehyde is the end-product of lipid peroxidation and is an effective marker for oxidative stress. Lipid peroxidation is a mechanism of cellular injury. Increased levels of this product have been associated with chronic disease including infections.

\section{Gut Histology Analysis}

Microscopic examination of the ilea in L. plantarum Dad-13 from both treatment and placebo groups showed no difference in normal condition. Subsequently, Fig. 3 shows that no inflammation had occurred. This result was supported by quantitative data analysis in gut morphology (Table 2). There were also no significant differences in villi height, epithelial height and mucosal thickness among all groups ( $>>0.05)$. Histological examination of intestinal and gut tissue was aimed at evaluating changes in mucosa integrity due to treatment. 
The results suggested that $L$. plantarum Dad-13 high dosage treatment did not affect the gut structure. Intestinal and gut barriers are the first defense mechanisms in the prevention of pathogen invasion of the blood. Feeding L. murinus to rats has shown alterations to the mucosal structure of the intestine causing bacterial translocation to the blood stream and organs (Ma et al., 2009). Invasion of Salmonella typhi could also cause changes to intestinal structure in rats.

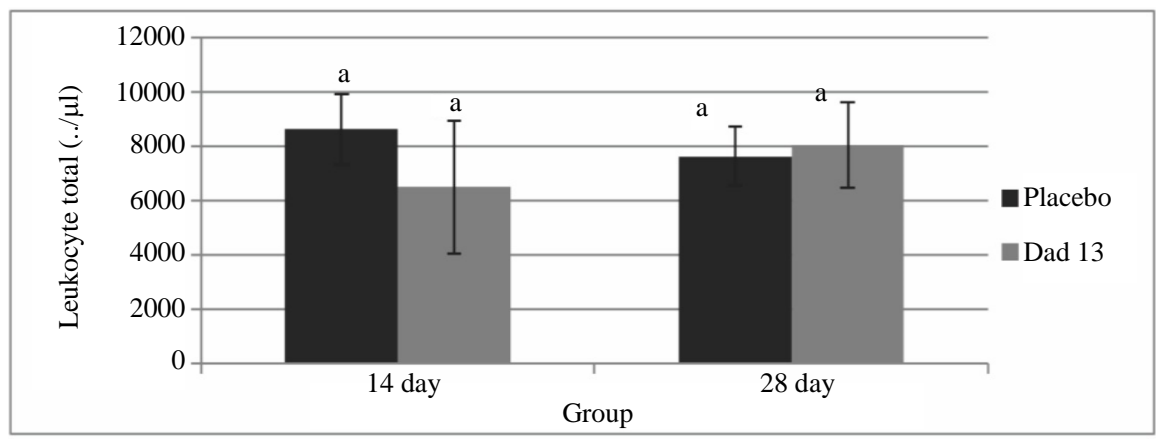

Fig. 1: Mean leukocyte totals $(. . / \mu 1)$ in male Sprague Dawley rats after high doses of L. plantarum Dad-13 treatment for 14 days and 28 days

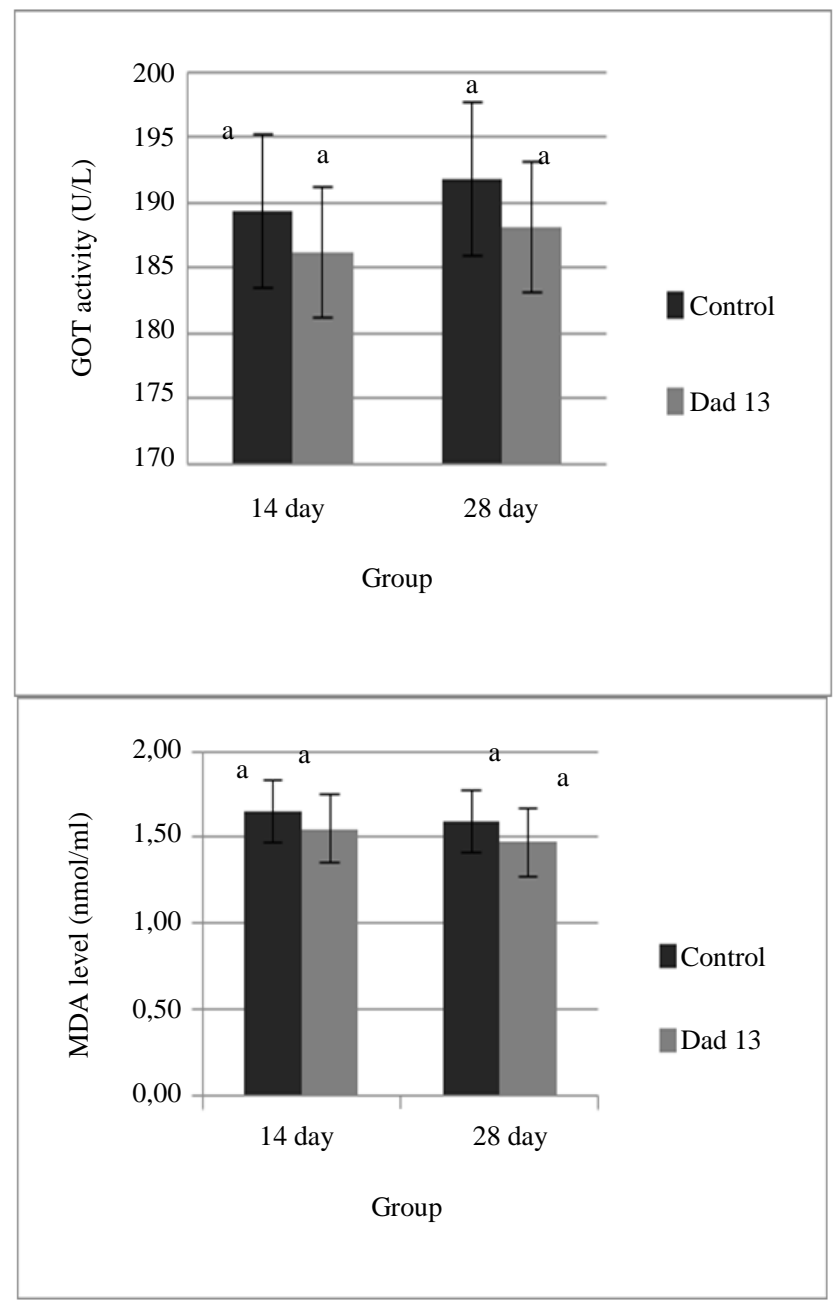

Fig. 2: Glutamic Oxalacetic Transaminase (GOT) Activity and Malondialdehyde (MDA) levels in male Sprague Dawley rats after treatment with high doses of L. plantarum Dad-13 for 14 days and 28 days 

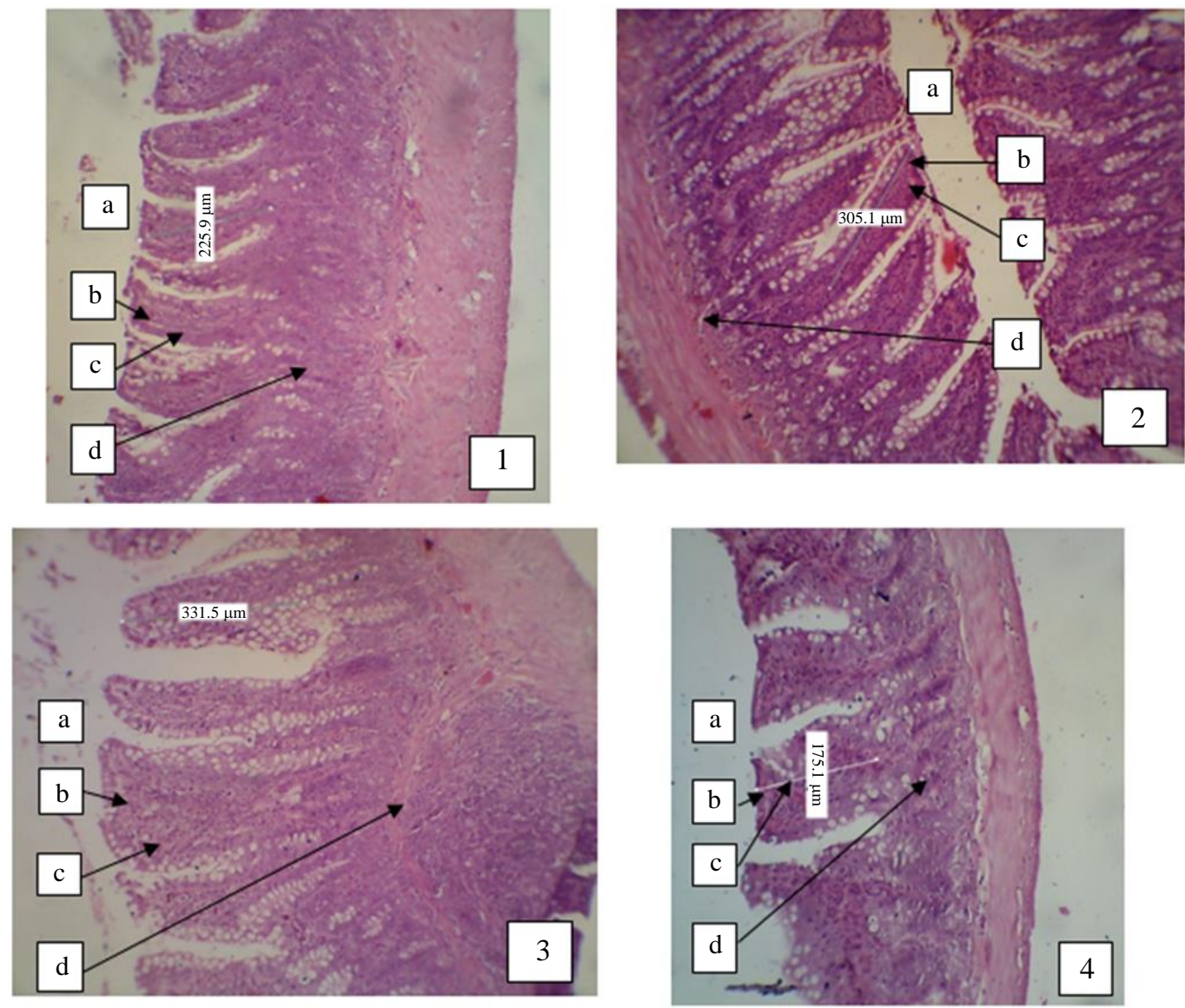

Fig. 3: Ileum microscopic appearance: (1) and (2) Control groups 14 and 28 days; (3) and (4) Treatment groups 14 and 28 days (notes: a. lumen; b. mucosa; c. muscularis mucosa; d. submucosa; H\&E stain)

Table 1: Leukocyte profile of male Sprague Dawley rats after high doses of L. plantarum Dad-13 treatment for 14 days and 28 days. Leukocyte

\begin{tabular}{|c|c|c|c|c|}
\hline \multirow[b]{3}{*}{ Profile } & \multicolumn{4}{|l|}{ Group } \\
\hline & \multicolumn{2}{|l|}{ 14-days } & \multicolumn{2}{|l|}{ 28-days } \\
\hline & Control & Treatment & Control & Treatment \\
\hline Neutrophyle (\%) & $46.00 \pm 6.00$ & $47.00 \pm 6,68$ & $41.00 \pm 7.83$ & $43.25 \pm 7.85$ \\
\hline Lymphocyte (\%) & $48.75 \pm 4.99$ & $47.00 \pm 4,00$ & $54.75 \pm 8.77$ & $54.5 \pm 5.80$ \\
\hline Monocyte (\%) & $5.25 \pm 3.50$ & $4.50 \pm 2.64$ & $4.25 \pm 1.89$ & $3.25 \pm 1.50$ \\
\hline
\end{tabular}

Table 2: Effect of high doses of L. plantarum Dad-13 for 14 days and 28 days on intestinal morphology of Sprague Dawley rats

\begin{tabular}{|c|c|c|c|c|c|}
\hline \multirow[b]{3}{*}{ Organ $(\mu \mathrm{n}$} & & \multicolumn{4}{|l|}{ Groups } \\
\hline & & \multicolumn{2}{|l|}{14 Days } & \multicolumn{2}{|l|}{28 Days } \\
\hline & & Placebo & Treatment & Placebo & Treatment \\
\hline \multirow[t]{3}{*}{ Ileum } & Villous height & $244.95 \pm 41.20$ & $216.32 \pm 101.9$ & $253.02 \pm 74.46$ & $249.37 \pm 128.9$ \\
\hline & Ephitel height & $21.4 \pm 3.13^{*}$ & $13.97 \pm 2.81$ & $16.3 \pm 2.49$ & $17.1 \pm 1.42$ \\
\hline & Mucosa thickness & $8.00 \pm 2.71$ & $9.25 \pm 6.07$ & $6.50 \pm 1.29$ & $5.75 \pm 0.95$ \\
\hline \multirow[t]{2}{*}{ Caecum } & Ephitel height & $17.9 \pm 4.07$ & $17.05 \pm 3.53$ & $16.58 \pm 2.49$ & $17.92 \pm 2.60$ \\
\hline & Mucosa thickness & $258.52 \pm 48.78$ & $259.76 \pm 60.0$ & $234.87 \pm 51.35$ & $229.01 \pm 21.3$ \\
\hline \multirow{2}{*}{ Colon } & Ephitel height & $17.57 \pm 4.28$ & $17.67 \pm 2.86$ & $18.45 \pm 3.09$ & $16.72 \pm 2.10$ \\
\hline & Mucosa thickness & $212.30 \pm 32.84$ & $198.50 \pm 17.0$ & $237.65 \pm 45.27$ & $217.95 \pm 45.9$ \\
\hline
\end{tabular}

* Superscript represents significant difference $(p<0.05)$ 
Populations of Lactic Acid Bacteria and $L$. plantarum in Feces of Rats

The populations of L. plantarum in the feces and digesta of the placebo group rats were very low and consumption of high dosage L. plantarum Dad-13 for 28 days markedly increased their numbers (Table 3 ). The results also showed that there was already a presence of lactic acid bacteria in placebo groups and the population was comparable to that of the treatment groups. It seems that lactic acid bacteria are commensal microflora of the gastrointestinal tracts of rats but not L. plantarum. These results suggested that $L$. plantarum Dad-13 could survive in the gastrointestinal tracts of rats. The ability to survive in the gastrointestinal tract is a probiotic criterion. Consumption of fermented milk containing $L$. plantarum Dad-13 by thirty healthy Indonesian volunteers resulted in significant increases in the numbers of $L$. plantarum in all volunteers' fecal specimens and decreases in the amounts of Enterobacteriaceae, E. coli and coliform on E coli in more than $50 \%$ of the volunteers' fecal specimens (Rahayu et al., 2016).

\section{Bacterial Analyses of Blood and Organs of Rats}

Bacterial analyses, using MRS media, of liver, spleen, kidney and blood of the rats showed that most samples contained lactic acid bacteria in both placebo and treatment groups (Table 4). Bacterial analyses using LPSM also showed bacteria colonies that could be $L$. plantarum in all samples. However, it is interesting that even in control group samples, lactic acid bacteria were also found in liver, spleen, kidneys and blood. It might be possible that the blood and organs of the rats already contained bacteria. A study reported that in translocation tests for L. rhamnosus $\mathrm{HN} 001$ and Bifidobacterium lactis in rat organs, lactic acid bacteria were found in a kidney sample (Shu et al., 2000a). However molecular analysis revealed that the bacteria did not emanate from the probiotic bacteria. Other research of probiotic safety assessments in rats also found the presence of lactic acid bacteria in organs (Lara-Villoslada et al., 2009; 2007; Shu et al., 2000a).

Another study stated that if translocation incidents occur similarly in an experiment on both control and treatment groups, it is not an indication that these occurrences were influenced by the probiotic treatment (Shu et al., 2000b). Furthermore, bacteria are occasionally contained in the mesenteric lymph nodes (MLN), liver and spleen of rats (Gordon et al., 1955). Indigenous gut bacteria of rats may translocate to the lymphoid tissues and be killed by the immune system in situ, as their presence can stimulate the immune system (Owens and Berg, 1992). Thus, further studies are necessary to investigate the bacteria in rat organs and blood and also their genetic relationship with $L$. plantarum Dad-13.

Sixteen isolates, taken from rat blood and organs, were able to grow on LPSM (Table 5). However, we are, as yet, unable to confirm that those bacteria came from the consumption of $L$. plantarum Dad-13. This is due to the fact that in the control group there were bacteria that can grow in LPSM. From those sixteen isolates, nine were Gram-positive rod cells and one a Gram-positive with coccus morphology. On the other hand there were also six Gram-negative bacteria types, which is indicative of non-lactic acid bacteria.

Table 3: Population of LAB and L. plantarum $\left(\log _{10} \mathrm{CFU} / \mathrm{g}\right)$ in rat digesta and feces using MRS and LPSM, after consuming high doses of L. plantarum Dad-13 for 28 days

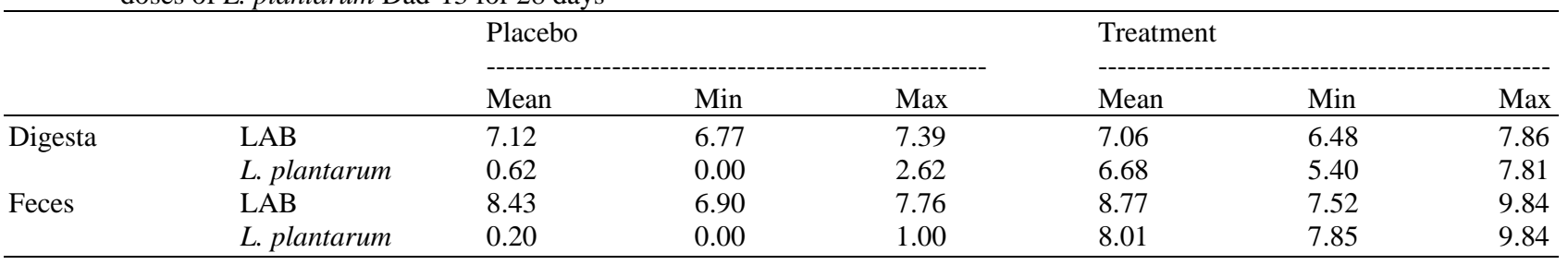

Table 4: Number of rats containing bacteria in blood and organs

\begin{tabular}{|c|c|c|c|c|c|c|c|c|c|c|}
\hline \multirow[b]{3}{*}{ Sample source } & \multirow{2}{*}{\multicolumn{2}{|c|}{$\begin{array}{l}\text { Initial } \\
\text { Control group }\end{array}$}} & \multicolumn{4}{|c|}{14 days treatment } & \multicolumn{4}{|c|}{28 days treatment } \\
\hline & & & \multicolumn{2}{|c|}{ Placebo group } & \multicolumn{2}{|c|}{ Treatment group } & \multicolumn{2}{|c|}{ Placebo group } & \multicolumn{2}{|c|}{ Treatment group } \\
\hline & MRS & LPSM & MRS & LPSM & MRS & LPSM & MRS & LPSM & MRS & LPSM \\
\hline Liver & $4 / 4$ & $0 / 4$ & $3 / 4$ & $1 / 4$ & $3 / 4$ & $2 / 4$ & $4 / 4$ & $0 / 4$ & $4 / 4$ & $4 / 4$ \\
\hline Spleen & $3 / 4$ & $2 / 4$ & $4 / 4$ & $2 / 4$ & $4 / 4$ & $3 / 4$ & $4 / 4$ & $1 / 4$ & $4 / 4$ & $4 / 4$ \\
\hline Kidney & $4 / 4$ & $0 / 4$ & $3 / 4$ & $1 / 4$ & $4 / 4$ & $0 / 4$ & $3 / 4$ & $1 / 4$ & $4 / 4$ & $4 / 4$ \\
\hline Blood & $1 / 4$ & $0 / 4$ & $2 / 4$ & $1 / 4$ & $3 / 4$ & $1 / 4$ & $3 / 4$ & $0 / 4$ & $4 / 4$ & $2 / 4$ \\
\hline
\end{tabular}

Number of rats containing bacteria/number of rats; MRS and LPSM: media for lactic acid bacteria and L. plantarum respectively 
Table 5: Bacteria isolated from mice organs and blood of treated and placebo samples

\begin{tabular}{|c|c|c|c|c|}
\hline \multirow[b]{2}{*}{ Sample source } & \multicolumn{2}{|c|}{14 days treatment } & \multicolumn{2}{|c|}{28 days treatment } \\
\hline & Control & Treatment & Control & Treatment \\
\hline Liver & 1 (CL3) & 1 (AL8) & $\mathrm{NI}$ & 2 (BL4,BL8) \\
\hline Spleen & $1(\mathrm{CS} 3)$ & $\mathrm{NI}$ & 1 (DS2) & 2 (BS3, BS4) \\
\hline Kidney & NI & NI & 1 (DK4) & $2(\mathrm{BK} 3, \mathrm{BK} 8)$ \\
\hline Blood & $1(\mathrm{CB} 2)$ & $1(\mathrm{AB} 9)$ & NI & 3 (BB1, BB2, BB 4$)$ \\
\hline
\end{tabular}

Note: NI (None Isolate)

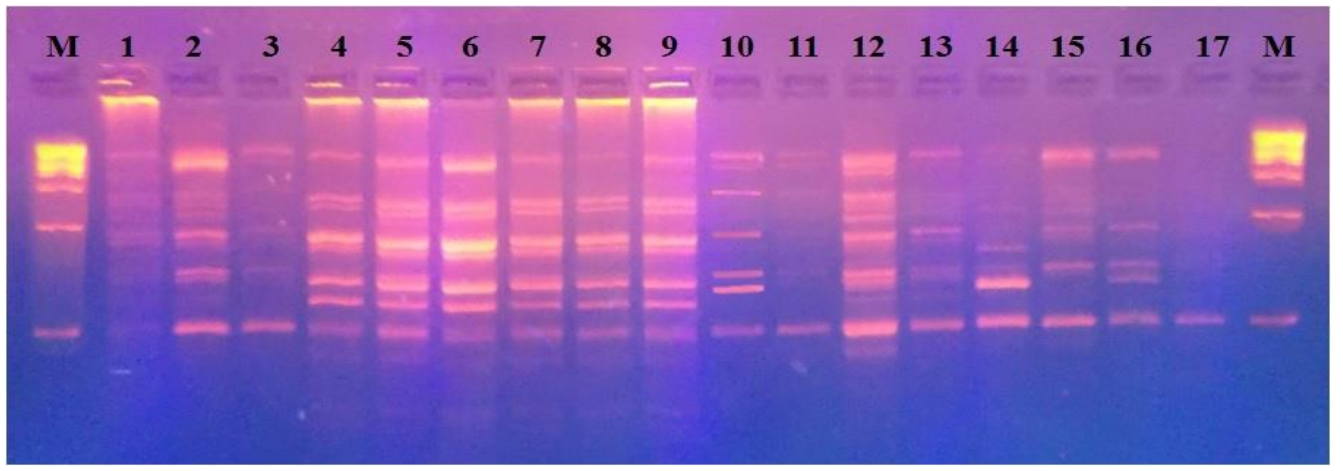

Fig. 4: Result of electrophoresis agarose gel 2\% of 16 isolates rep-PCR derived from L.plantarum Dad-13 treated rats (AL8, AB9, BB1, BB2, BB4, BK3, BK8, BL4, BL8, BS3, BS4) and non-treated (CB2, CL3, CS3, DK4, DS2) rats. (Lane M: Marker; 1:BS3; 2:BS4; 3:CS3; 4:DS2; 5:AB9; 6:BB1; 7:BB2; 8:BB4; 9:CB2; 10:L. plantarum Dad-13; 11:BK3; 12:BK8; 13:DK4; 14:AL8; 15:BL4; 16:BL8; 17:CL3.)

However, a study reported that Enterococcus faecalis can use sorbitol as a source of energy and produce lactic acid from it (Holt et al., 1994). Moreover, based on another study, E. faecalis is resistant to the antibiotic ciprofloxacin contained in LPSM (Lee, 2013). It is possible that the bacteria found in the blood and organs of the rats were not a result of the consumption of high doses of L. plantarum Dad-13. It could be other bacteria, such as E. faecalis, found in the blood and organs of rats.

\section{Identifying the Rep-PCR Bacteria using BOX AlR Primer}

For further identification of the type of bacteria grown in LPSM and isolated from the blood and organs of rats, further analyses were continued using genotypic or molecular methods. Thus, it could be used to distinguish the strain of $L$. plantarum Dad-13 from the 16 isolates obtained from organs and blood of the control and treatment groups. Repetitive-Polymerase Chain Reaction (rep-PCR) is a fingerprinting method used to differentiate between strains of lactic acid bacteria, such as Lactobacillus johnsonii (Ventura and Zink, 2002) and Bifidobacteria, by using BOX A1R primer (Masco et al., 2003).

The results of rep-PCR for 16 isolates were compared with L. plantarum Dad-13 (Fig. 4). The visualization of agarose gel electrophoregram showed that all 16 isolates have different DNA repetitive patterns which also differ from that of L. plantarum Dad-13. Variations in the DNA repetitive pattern on agarose gel indicated different bacterial levels and strains (Masco et al., 2003). The matrix data of the pattern was then processed using software NTSYSpc 2.1 in Sequential Agglomerative Hierarchical Nesting (SAHN), as had been carried out in previous research, to obtain the dendogram (Versalovic et al., 1994). The genetic relationships of those 16 isolates are shown in Fig. 5. The isolate with the highest similarity registered $93 \%$ and the lowest $71 \%$. These were then processed on genetic analysis by $16 \mathrm{SrRNA}$.

To examine the genetic relationship between 16 isolates from the blood and organs of rats and isolates from genebank NCBI, a phylogenetic tree was developed using MEGA 5.0 software (Tamura et al., 2011). The result derived from the phylogenetic tree, as seen in Fig. 6, showed that L. plantarum Dad-13 has a distant genetic relationship with the other isolates from the organs and blood of the rats in both the control and treatment groups. The isolate with the highest similarity to $L$. plantarum Dad-13 in the rep-PCR analysis was BK3. However, in the phylogenetic tree, gen $16 \mathrm{~S}$ rRNA was located in a different branch from L. plantarum Dad-13. Isolates of BL8, BL4 and DK4 were also located in different groups from $L$. plantarum Dad-13. The isolate which had the most distant genetic relationship with $L$. plantarum Dad-13 was CL3. CL3 has a close genetic relationship with Enterococcus faecalis. 


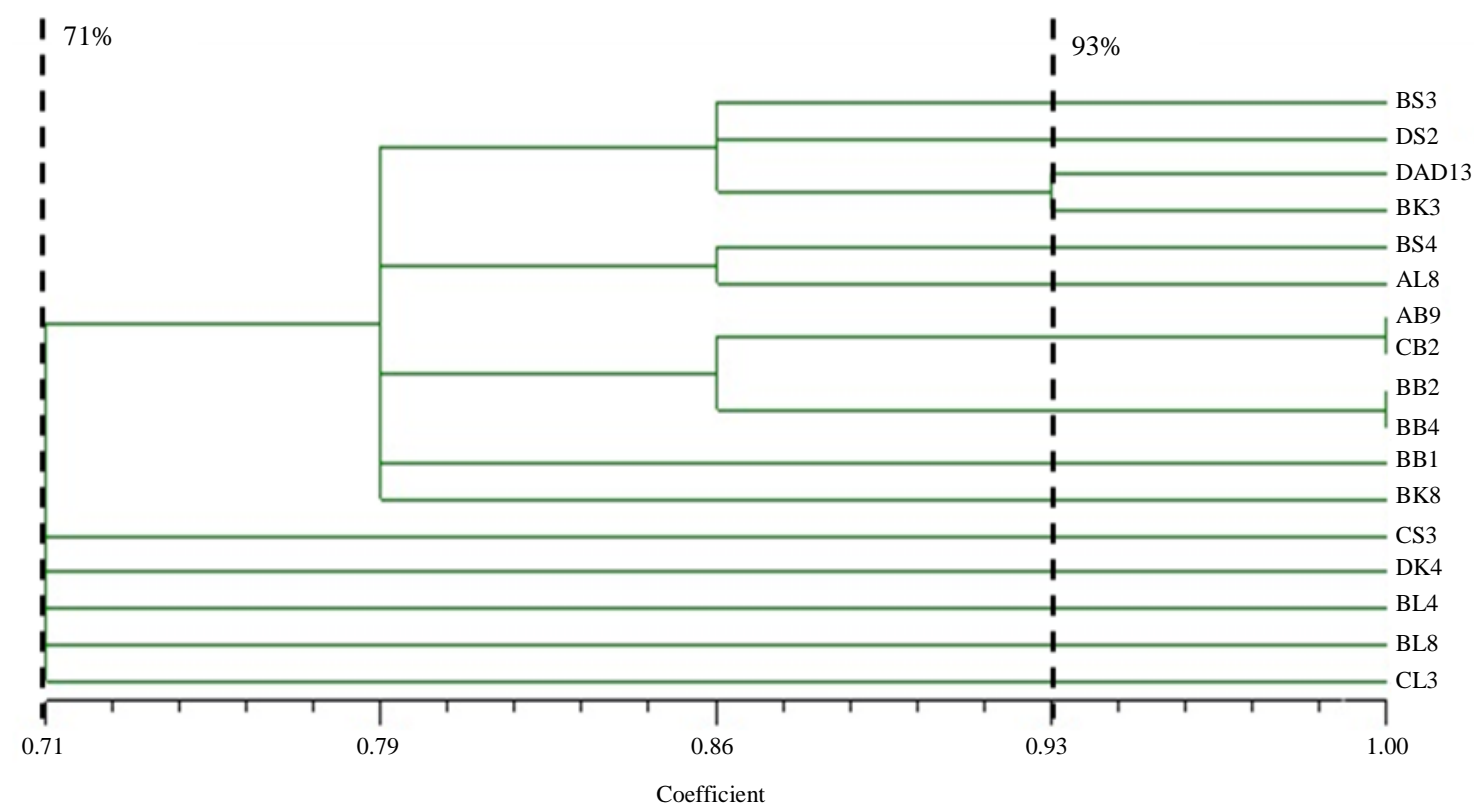

Fig. 5: Dendogram of 16 bacterial isolates from rats shows the lowest (71\%) and the highest (93\%) similarity to L. plantarum D13

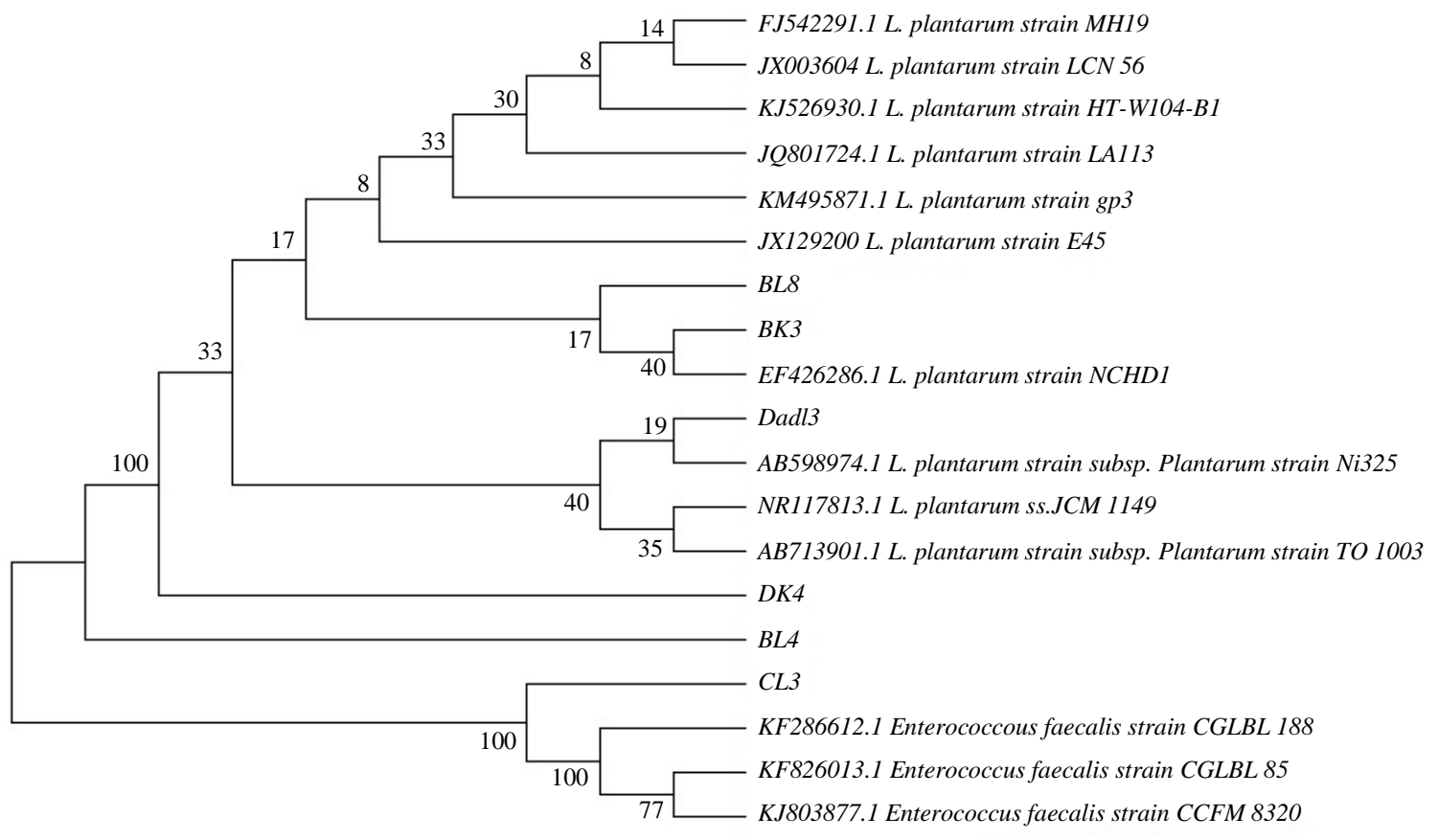

Fig. 6: The genetic relationships among the isolates derived from the organs and blood of rats treated with L. plantarum Dad-13 (BK3, BL4 and BL8) and control (CL3 and DK4) with several species based on the gene 16S rRNA gene sequence. The numbers in this phylogenetic tree show the percentage of bootstrap value from 1000 repetitions

An interesting point from the result of identification using the $16 \mathrm{~S}$ rRNA method is that there is a non- L. plantarum bacterium which can grow in differential selective medium of LPSM. This is due to the ability of E. faecalis to use sorbitol as a source of energy and produce lactic acid from it (Holt et al., 1994). E. faecalis is resistant to antibiotic ciprofloxacin contained in LPSM. This means that $E$. 
faecalis will still live in any medium containing antibiotic ciprofloxacin and have almost the same appearance as L. plantarum (Lee, 2013). From the molecular analyses, it can be confirmed that bacteria found in the blood and organs of rats are different from L. plantarum Dad-13 and were not caused by the consumption of high doses of L. plantarum Dad-13.

\section{Conclusion}

The results of this study show that consumption of high doses $\left(10^{11} \mathrm{CFU}\right)$ of Lactobacillus plantarum Dad13 for 28 days in Sprague Dawley rats had no deleterious effect on the rats' performance (feed intake, body weight, hematological concentration, physiological and stress markers and gut morphology). L. plantarum Dad13 can survive in gastrointestinal tract of rats, resulting in a significant increase of the $L$. plantarum population in the fecal matter and the digesta of treated rats. It can be concluded that consumption of high doses of $L$. plantarum Dad-13 for 28 days did not cause bacterial translocation in the organs or blood of the rats. Although this study was limited to the use of animal study, the findings are useful to support the safety assessment of the use of L. plantarum Dad-13 as a probiotic.

\section{Acknowledgement}

This work was supported by the Ministry of Research, Technology and Higher Education of the Republic of Indonesia.

\section{Author's Contributions}

Endang S. Rahayu: Conceived, designed and supervised the research.

Ilzamha H. Rusdan: Carried out the experiments.

Armita Arthennia: Performed data analysis.

Rafli Z. Kamil and Putrika C. Pramesi: Contributed in writing the manuscript.

Yustinus Marsono: Supervised biomolecular analysis.

Tyas Utami and Jaka Widada: Supervised microbiology analysis.

\section{Conflict of Interest}

The authors declare no conflict of interest.

\section{References}

Albro, P.W., J.T. Corbett and J.L. Schroeder, 1986. Application of the thiobarbituric assay to measurement of lipid peroxidation products in microsomes. J. Biochem. Biophys. Meth., 13: 185-194. DOI: $10.1016 / 0165-022 \mathrm{X}(86) 90092-8$
Ausubel, F., R. Brent, R. Kingston and D. Moore, 2002. Short Protocols in Molecular Biology. 5th Edn., John Wiley and Sons Publication, New York.

Bujalance, C., M. Jiménez-valera, E. Moreno and A. Ruiz-bravo, 2006. A selective differential medium for Lactobacillus plantarum. J. Microbiol. Meth., 66: 572-575. DOI: 10.1016/j.mimet.2006.02.005

Donohue, D., 2006. Safety of probiotics. Asia Pac. J. Clin. Nutr., 15: 563-569. DOI: 10.1159/000345744

FAO/WHO, 2002. Guidelines for the evaluation of probiotics in food. Rep. a Jt. FAO/WHO Work. Gr. Draft. Guidel. Eval. Probiotics Food London, On.

Gordon, B.Y.L.E.E.E., P.D., Ruml, H.J.O.D., Hahne and C.P. Miller, 1955. Studies on susceptibility to infection following ionizing radiation, Chapter IV: The Pathogenesis of the Endogenous Bacteremias in Mice. Am. Soc. Microbiol. J.

Holt, J., N. Krieg, P.H. Sneath, J. Staley and S. Williams, 1994. Bergey's Manual of Determinative Bacteriology. 9th Edn., Williams and Wilkins, Maryland, USA.

Kinnebrew, M.A. and E.G. Pamer, 2012. Innate immune signaling in defense against intestinal microbes. Immunol. Rev., 245: 112-131. DOI: 10.1038/ng.3564.Distinct

Lara-Villoslada, F., S. Sierra, M.P. Díaz-Ropero, J.M. Rodríguez and J. Xaus et al., 2009. Safety assessment of lactobacillus fermentum CECT5716, a probiotic strain isolated from human milk. J. Dairy Res., 76: 216-221.

DOI: $10.1017 / \mathrm{S} 0022029909004014$

Lara-Villoslada, F., S. Sierra, R. Martín, S. Delgado and J.M. Rodríguez et al., 2007. Safety assessment of two probiotic strains, Lactobacillus coryniformis CECT5711 and Lactobacillus gasseri CECT5714. J. Applied Microbiol., 103: 175-184. DOI: $10.1111 / \mathrm{j} .1365-2672.2006 .03225 . \mathrm{x}$

Lee, G., 2013. Ciprofloxacin resistance in enterococcus faecalis strains isolated from male patients with complicated urinary tract infection. Korean J. Urol., 54: 388-393. DOI: 10.4111/kju.2013.54.6.388

Ma, L., E. Deitch and R. Specian, 2990. Translocation of Lactobacillus murinus from the gastrointestinal tract. Curr. Microbiol., 20: 177-184.

DOI: 10.1007/BF02091994

Masco, L., G. Huys, D. Gevers, L. Verbrugghen and J. Swings, 2003. Identification of bifidobacterium species using rep-PCR fingerprinting. Syst. Applied Microbiol., 26: 557-563. DOI: $10.1078 / 072320203770865864$

Owens, W.E. and R.D. Berg, 1992. Bacterial translocation from the gastrointestinal tract. J. Med., 23: 217-244. 
Rahayu, E.S., 2003. Lactic acid bacteria in fermented foods of Indonesian origin. agriTECH, 23: 75-84. DOI: $10.22146 /$ agritech.13515

Rahayu, E.S., M. Cahyanto, L. Windiarti, J. Sutriyanto and T. Kandarina et al., 2016. Effects of consumption of fermented milk containing indigenous probiotic lactobacillus plantarum dad-13 on the fecal microbiota of healthy Indonesian volunteers. Int. J. Probiot., 11: 91-98.

Reeves, P.G., F.H. Nielsen and G.C. Fahey, 2018. AIN93 purified diets for laboratory rodents: Final report of the American institute of nutrition ad hoc writing committee on the reformulation of the AIN-76A rodent diet. J. Nutr. 123: 1939-1951.

DOI: $10.1093 / \mathrm{jn} / 123.11 .1939$

Salminen, M.K., H. Rautelin, S. Tynkkynen, T. Poussa and M. Saxeli et al., 2006. Lactobacillus bacteremia, species identification and antimicrobial susceptibility of 85 blood isolates. Clin. Infect. Dis., 42: e35-e44. DOI: 10.1086/500214

Salminen, S., A. von Wright, L. Morelli, P. Marteau and D. Brassart et al., 1998. Demonstration of safety of probiotics - A review. Int. J. Food Microbiol., 44: 93-106.

Shu, Q., J. Zhou, K. Rutherfurd, J. Prasad and M. Birtles et al., 2000a. Safety assessment of potential probiotic lactic acid bacterial strains Lactobacillus rhamnosus HN001, Lb. acidophilus HN017 and Bifidobacterium lactis HN019 in BALB/c mice. Int. J. Food Microbiol., 56: 87-96.

DOI: $10.1016 / \mathrm{S} 0168-1605(00) 00219-1$
Shu, Q., J. Zhou, K. Rutherfurd, J. Prasad and P. Gopal et al., 2000b. Acute oral toxicity and bacterial translocation studies on potentially probiotic strains of lactic acid bacteria. Food Chem. Toxicol., 38: 153-161. DOI: 10.1016/S0278-6915(99)00154-4

Snydman, D.R., 2008. The safety of probiotics. Clin. Infect. Dis., 46: S104-S111. DOI: 10.1086/523331

Tamura, K., D. Peterson, N. Peterson, G. Stecher and M. Nei et al., 2011. MEGA5: Molecular evolutionary genetics analysis using maximum likelihood, evolutionary distance and maximum parsimony methods. Mol. Biol. Evol., 28: 2731-2739.

DOI: $10.1093 / \mathrm{molbev} / \mathrm{msr} 121$

Ventura, M. and R. Zink, 2002. Specific identification and molecular typing analysis of Lactobacillus johnsonii by using PCR-based methods and pulsed-field gel electrophoresis. FEMS Microbiol. Lett., 217: 141-154. DOI: 10.1016/S0378-1097(02)01070-4

Versalovic, J., G.M. Schneider, F. Bruijn and J.R. Lupski, 1994. Genomic fingerprint of bacteria using repetitive sequence-based polymerase chain reaction. Meth. Molecular Cellular Biol., 5: 25-40.

Wardani, S.K., M.N. Cahyanto, E.S. Rahayu and T. Utami, 2017. The effect of inoculum size and incubation temperature on cell growth, acid production and curd formation during milk fermentation by Lactobacillus plantarum Dad 13. Int. Food Res. J., 24: 921-926. 